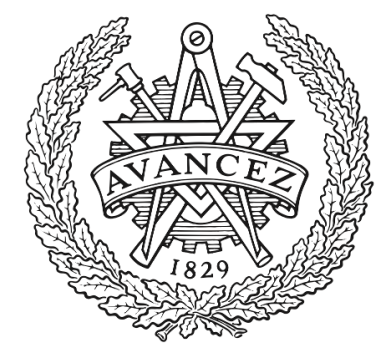

CHALMERS

UNIVERSITY OF TECHNOLOGY

\title{
instance: Soma-based multi-user interaction design for the telematic sonic arts
}

Downloaded from: https://research.chalmers.se, 2023-04-26 13:36 UTC

Citation for the original published paper (version of record):

Strauss, L., Tatar, K., Nuro, S. (2021). instance: Soma-based multi-user interaction design for the telematic sonic arts. Organised Sound, 26(3): 390-402.

http://dx.doi.org/10.1017/S1355771821000479

N.B. When citing this work, cite the original published paper. 


\title{
instance: Soma-based multi-user interaction design for the telematic sonic arts
}

\author{
LUCY STRAUSS ${ }^{1}$, KIVANÇ TATAR ${ }^{2}$ and SUMALGY NURO ${ }^{3}$ \\ ${ }^{1}$ University of British Columbia,Vancouver, Canada. Email: lucystrauss@protonmail.com \\ ${ }^{2}$ Chalmers University of Technology, Gothenburg, Sweden. Email: tatar@chalmers.se \\ ${ }^{3}$ University of Cape Town, Cape Town, South Africa. Email: sumalgyjafar@gmail.com
}

\begin{abstract}
The telematic work instance is a performance for viola and dance that digitally connects performers in Vancouver and Cape Town. The network interface enables a violist and a dancer to simultaneously play multi-user digital music-dance instruments over the internet with music and dance. The composition, design and performance interaction of instance draw from acoustic multi-user instrument paradigms and music-dance interactions in the African performing arts to explore the idiosyncrasies of the telematic performance space. The iterative design process implements soma-based research methods to inspire sonic compositional material with the body and to explore the performers' embodied experience of sonic aesthetics during their interaction.
\end{abstract}

\section{INTRODUCTION}

The live-streamed, telematic work instance was created for a musician and a dancer, featuring elements of composition constructed from the sonic worlds of mbira and viola. It grew from the need for a real-time, collaborative performance practice during COVID-19 lockdowns and travel restrictions in 2020, and was presented in a live-streamed performance on 30 August 2020 (instance 2020) with performers situated in Cape Town (South Africa) and Vancouver (Canada).

Our motivations include exploring telematic and interactive music-dance possibilities within the design of multi-user digital music-dance instruments (DMDIs) (detailed in section 2.3) beyond bi-directional audiovideo streaming. We engage with principles and philosophical and somatic concepts in the body of knowledge on African music to conceptualise sonic composition for multi-user instrument design and musicdance interaction in the digital performance space.

Much research in telematic performance is dedicated to studying the embodied experience of performers in the telematic performance space (Lemmon 2019). Perceptual shift is a central concept in many musical practices on the African continent, such as the mbira practices in Mozambique and Zimbabwe (Berliner 2020) and the Kiganda amadinda xylophone practice (discussed in section 2.2). We approach perceptual shift in music dance performance as an embodied experience and a lens through which to study performers' experience in telematic performance, where each performer's temporal experience is unique due to network latency. We use the body as a starting point to draw sonic possibilities in the artistic process, utilising somaesthetic and soma-design principles in the composition and design processes of instance.

\section{BACKGROUND}

The telematic work instance brings together the areas of multi-user instruments, network performance and musicdance instruments. Our approach to telematic performance takes inspiration from multimodal paradigms and performance concepts in the African performing arts. In the following sections, we cover the background of these areas and make reference to related works.

\subsection{Digital multi-user instruments and network performance}

The interactive dance system in the telematic framework of instance (detailed in section 3.2) provides an interface for the performers to play a multi-user digital musical instrument (DMI) in the telematic performance space, drawing from the tradition of DMI design in networked performance. The histories of DMIs and networked performance are inextricably linked (Jordà 2005). John Cage's Imaginary Landscapes No. 4 (1951) for 24 performers on 12 radios (Cage 1960) was one of the first networked music performances (Muller, Burger, Giovanni and Ziegler 2019) and an example of an electronic multi-user instrument (Jordà 2005). Bringing Cage's concepts into the digital realm, the League of Automatic Composers performed with the idea that their network was both one multi-user instrument and an ensemble of individual instruments (Bischoff, Gold and Horton 1978). Similarly, instance incorporates a network interface and the sonification of multiple performer inputs, existing as both a networked performance and a multi-user instrument.

\subsection{Multi-user instruments and multimodal interaction paradigms}

Existing in the telematic performance space, the DMI in instance embraces perceptual shift and is 
conceptually and experientially similar to acoustic paradigms in the African performing arts. For example, the interaction between musicians playing from opposite sides on the same xylophone, or the interaction between a dancer and drummer in moment-to-movement improvisation. These interactions illustrate the concepts of flow and agency in relation to the felt-experience of the artists.

Interlock is a central concept in many musical practices in Africa (Agawu 2014), such as the mbira practices of Mozambique and Zimbabwe. Mbira features prominently in instance, as detailed in sections 4 and 5. In traditional mbira practice, the player composes a quick, cyclical pattern in constant pulsation. An underlying melody weaves through the pattern, interlocking between the notes played with the left and right hands. Skilled mbira players improvise or compose variations on the original pattern, to bring out different elements of the melody (Berliner 2020). A composition can be played for hours at a time. Because of the cyclical nature, those who are listening or playing along could experience the melody in any number of ways, depending on their felt perception of its starting point.

Perceptual shift, also referred to as simultaneous multidimensionality (Locke 2009), is evident in many African music practices. For example, perceptual shift resulting from interlock in a multi-user instrument context is evident in the Kiganda amadinda xylophone practice. One musician starts to play a melody in regular pulsation. A second player sits on the opposite side of the instrument and joins with another melody, in constant pulse, falling halfway between the first player's pulses in time. The combination of the two interlocking parts forms a melody. A third player joins in the upper register of the same instrument, doubling this resulting melody (Kubik 1969). The first two musicians experience the beat in exactly opposite temporal locations rather than as syncopation, like one might in Western-based music practices. This embodied experience determines their perception of each individual component of the music and the performers' awareness of the combined sonic output. Playing a single instrument, the felt and sonic experience of performers are contributions to a shared aesthetic output, and there is no single absolute way to experience the music in time.

In a similar manner, the interplay of drummer and dancer in the Baganda Baakisimba practice expresses a dynamic give and take where both performers lead and follow, cueing each other in both deliberate and unintended synchronicities and juxtapositions. As much as the musician, the dancer defines and shapes the aesthetic moment through an improvisatory performance practice (Nannyonga-Tamusuza 2015).

The elements of perceptual shift emerge from the organic principle of interlock, the dancer's significant agency over a shared sonic output, and improvisation towards unintended synchronicities and juxtapositions. These elements comprise an accurate model for the multi-user DMDI and music-dance interactions in instance. We incorporated these embodied concepts into our composition, rehearsals and performance, using soma-based design strategies to explore the temporal separation (Chafe, Cáceres and Gurevich 2010) caused by network latency.

\subsection{Digital music-dance instruments}

The Digital Dance and Music Instrument (DDMI) is a conceptual tool for the design framework of a digital music instrument (DMI) played through dance (Tragtenberg, Calegario, Cabral and Ramalho 2019). We use the term 'digital music-dance instrument' instead because our approach has roots in music-dance practice. As a combination of a DMI and an interactive music system (Tatar and Pasquier 2019), the concept of the DMDI enables the designer to integrate both an instrumental relationship with music and a bodily expressiveness with equal importance into the design. Though not explicitly designed as a DMDI, another example of a multi-user music-dance instrument is the 'body-machine instrument' in Vrengt (Erdem, Schiand Jensenius 2019), where the musician alters the sonification of the dancer's movement. Hereby, the performers are focused on both movement and sound, playing the system together as a single instrument. In instance, the multimodality of music-dance performance fosters bidirectional sonic agency between musician and dancer through a multi-user telematic instrument. Similarly to the sonic agency of the dancer in Baakisimba improvisation practice (detailed in section 2.2), the dancer in instance plays the DMDI, moving with the intention of altering the shared sonic output.

\section{TECHNICAL FRAMEWORK DESIGN}

In the following section, we outline the motivations that led us to design specifications of the DMDIs in instance. Then, we describe the technical details of the telematic framework that facilitated our exploration of multimodal interaction possibilities beyond an audiovideo stream.

\subsection{Design motivations and specifications}

Our design motivations are influenced by the limitations of social-distancing during the COVID-19 pandemic. The dancer had to rehearse and perform from home in complete isolation under the South African national lockdown restrictions, with no inperson technical assistance. Hence, we aimed for a technical framework with a simple technology set-up on the dancer's side, running all complex technologies on the violist's side. 
Beyond the framework of an audiovideo stream of a networked performance, we were curious to explore multimodal interaction possibilities of music-dance performance in a telematic framework and to express the philosophical qualities of telematic performance through sound and movement through the design of DMDIs.

The performers' experience of flow was an important consideration in our design process. We centred the DMDI designs around resemblance to familiar performance instruments to minimise the impact of the technical constellation on flow, deciding that neither performer should physically touch the interface beyond their instrument of expression to play the DMDIs. Rather, synthesis parameters are controlled through mapping of body-tracking data and viola audio features, towards a representation of familiar instruments in the multi-user DMDI controls.

\subsection{Telematic framework}

We first needed a technical framework that satisfied the design specification of simple technology on the dancer's side, to facilitate our iterative design processes of DMDIs. Conceptually, the telematic framework is designed in such a way that neither performer touches the interface beyond their own body or acoustic musical instrument, bringing to light the intangible and ephemeral qualities of music within the space and time of the telematic performance environment.

Next, we explored a signal processing framework to be implemented on the musician's laptop. We used Max MSP for body-tracking and audio signal data processing, and utilised TouchDesigner for routing video data over NDI (Figure 1). We used a screencapture of the Zoom call to input the dancer's video stream to TouchDesigner (pipeline 1 in Figure 1), then routed this video stream to Max MSP using NDI (pipeline 2). A Max patch hosted an implementation of PoseNet - the n4m-posenet (Yogo 2021) Max external that connects to the PoseNet browser-based demo using Node for Max. Using the dancer's video stream, PoseNet tracked the dancer's body (Figure 2), which is one of two inputs of the multi-user DMDI.

Viola audio signal is the second input. The signal processing of viola audio is a real-time audio feature extraction using the Max MuBu (Schnell, Röbel, Schwarz, Peeters and Borghesi 2009) and PiPo externals (Schnell, Schwarz, Larralde and Borghesi 2017). The implementation is available as open-source (Tatar 2021). Our feature set includes bow velocity and acceleration, pitch detection and loudness. We used the fzero Max object to detect pitch and the loudness calculation is in digital $\mathrm{dB}$ scale. Although we tried the YIN algorithm (de Cheveigné and Kawahara 2002) using PiPo to detect the pitch, we observed that fzero was more consistent for the low pitch register of the viola. The bow velocity calculation is a simplification of the technique presented by Pardue, Buys, Edinger, Overholt and McPherson (2019). We first take the absolute of the viola signal, then apply a sample by sample integration with a window size of two samples. Finally, we add a lowpass filter with $79.2 \mathrm{~Hz}$ cutoff frequency. From this result, we calculate the acceleration from the first order differential of the velocity signal. Mapping these features to the synthesis parameters creates a pipeline for sonification of viola gestures within the multi-user DMDI.

We routed a mix of viola sound and synthesised audio from Max into the Zoom call with Soundflower (pipeline 3 in Figure 1) and selected the 'use original audio' feature on Zoom. We streamed the performance to YouTube with a Zoom Pro account (pipeline 4).

\section{COMPOSITION AND DESIGN}

The technical design of the DMDIs facilitates interaction with the sonic material in instance. Therefore, the design and composition processes are interwoven. In the following section, we discuss conceptualisations on temporality in the telematic performance space. Next, we introduce our methodology and detail the composition process, which was carried out using soma-based strategies through a Research through Design (RtD) approach to draw sonic possibilities from the body. The conceptualisations inspire the choice of sonic and movement materials and how they are used in the composition process.

\subsection{Space and time in compositions for telematic performance}

Müller et al. (2019) discuss fragmentation of the timespace continuum in telematic performance, through digitalisation, reflecting on the continuous and the discrete, and the reordering of events. Telematic performance is deconstructed from the dimensions of reality in the continuous medium of spacetime where the performers exist, then reconstructed in another physical location from discrete data. A symbolic juxtaposition can be drawn between the acoustic instruments that provide the sonic materials in instance. The viola can play continuously variable pitches and is evocative of the analogue. The mbira creates only discreet pitches and evokes the digital.

In his philosophical writings on time and reality, Rovelli (2019) proposes that we assume time flows continuously because we are unable to distinguish the smallest temporal intervals as a result of our inability to escape subjective reality - the limitations of the human interface with space-time. Inspired by Rovelli's proposition, we centred our artistic 


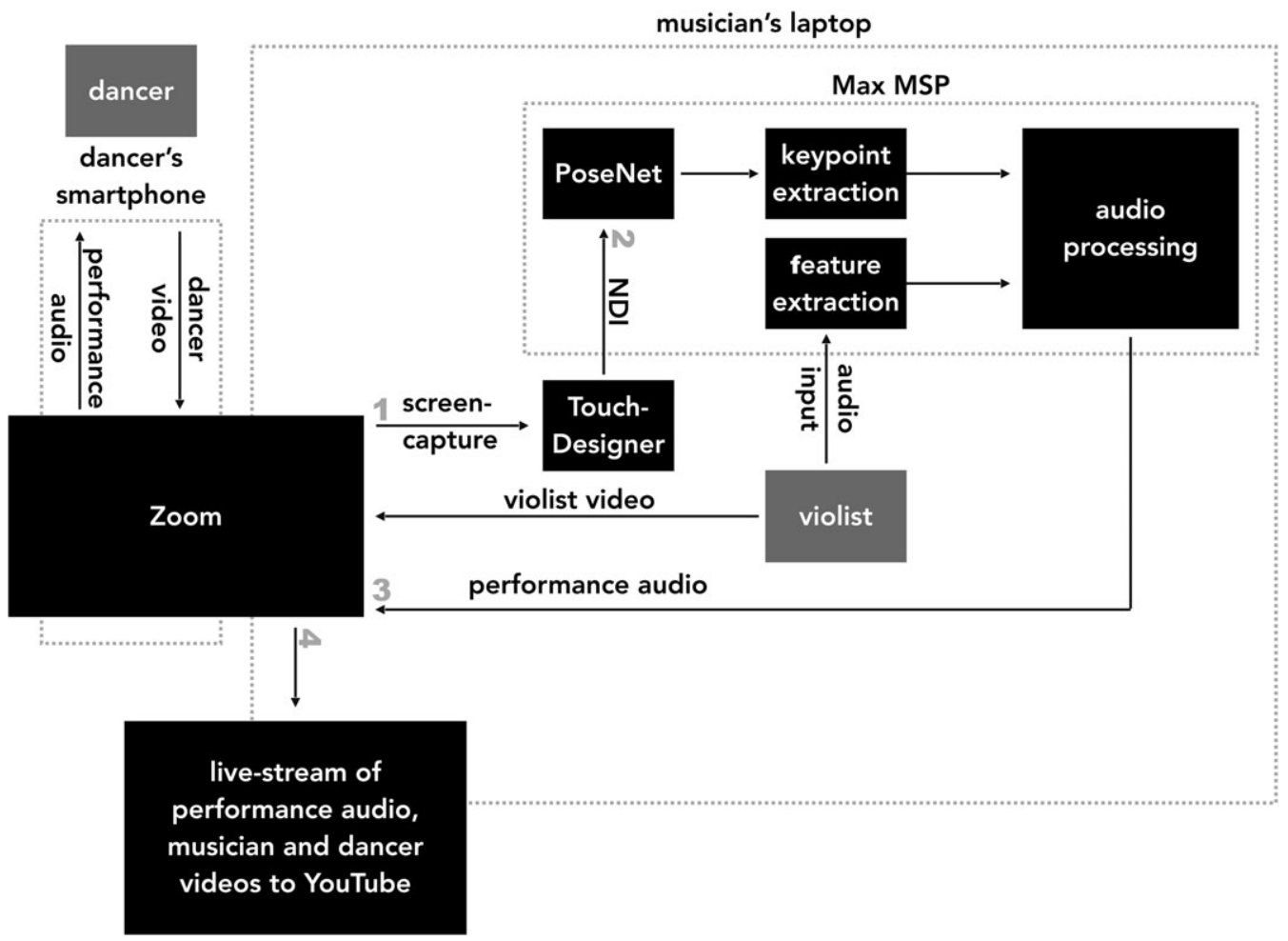

Figure 1. Telematic framework of instance.
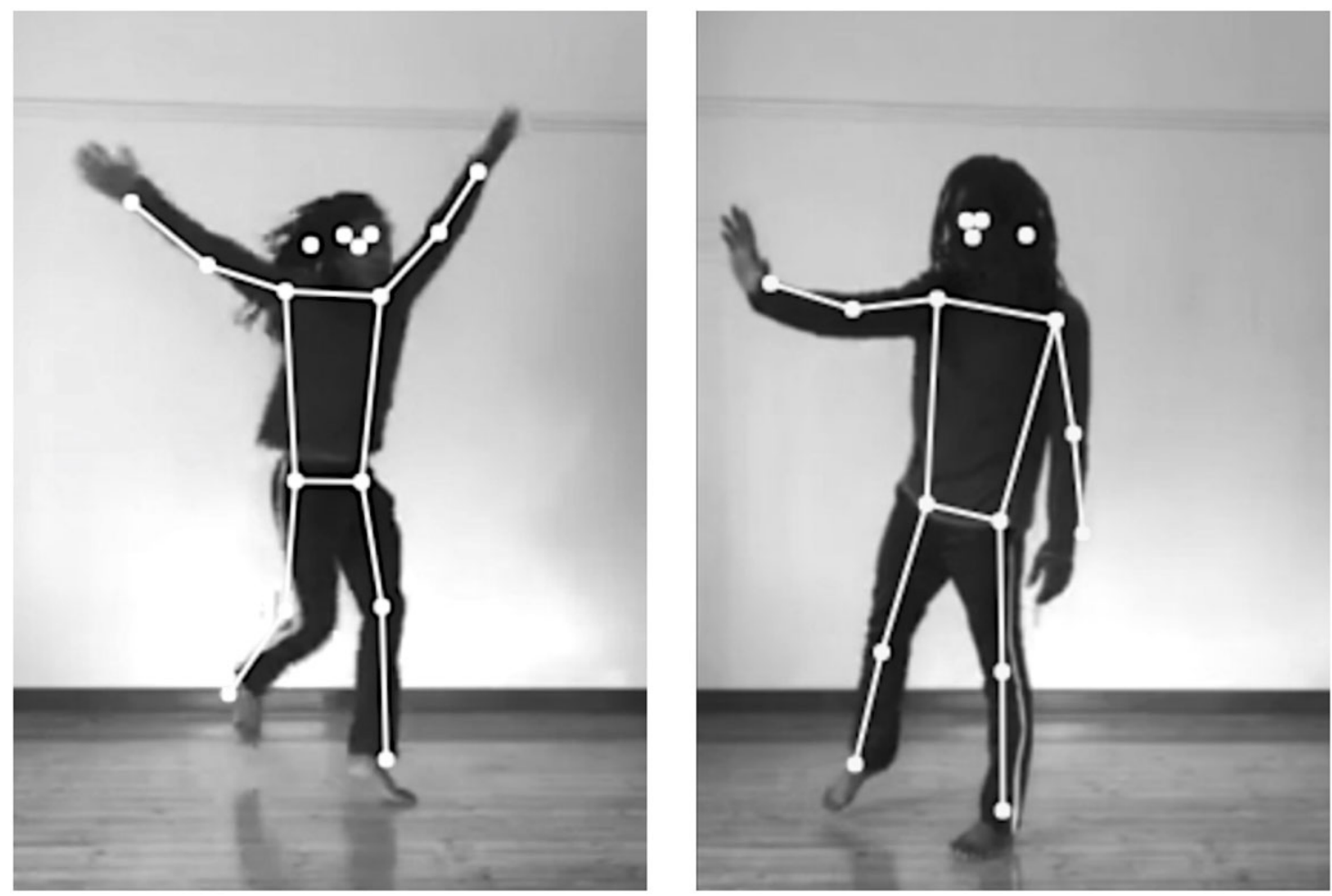

Figure 2. Body tracking using PoseNet. 
exploration of the temporal limitations of the telematic performance space within the human body itself as our most direct interface with space-time. During this exploration (detailed in section 4.2), we further explored how this proposition relates to the telematic interface: since temporal continuity has only been assumed, it is possible that a digital telematic interface captures and re-orders events between discrete dimensions, rather than fragmenting a continuous reality through digitalisation. Almost 100 years ago, Schoenberg first justified atonality with the 'emancipation of the dissonance' (Schoenberg 1952). Telematic performance enables the emancipation of performance from the order of time, offering creators of telematic performances an opportunity to perform with subjective temporality.

Performers' embodied experience of time is important in the study of performance excellence and musical expressivity, even outside the telematic medium. Mental imagery can allow performers to 'feel the musical process' (Stachó 2018), towards an embodied performance practice, while a distortion in the perceived experience of the passing of time is an indicator of flow (Araújo and Hein 2019). Similar to the perceptual shift in African music practices, a performer's ability to shift their attention forward and backward in time on any time scale through a mental navigation process is associated with performance excellence (Stachó 2018). Deliberate focus on mental navigation during performance is often associated with visio-spatio and gestural imageries (ibid.). For this reason, we started the iterative design process for instance from the body, using mental imagery to explore movement qualities the telematic performance space might evoke.

In network and telematic performance research, the internet is an acoustic and musical medium (Chafe 2009) and a performance space (Lemmon 2019). One way that performers can adjust their qualities of expression to better suit the telematic performance space is to anticipate larger rather than smaller intervals of time. In a network music study focusing on latency, researchers observed that musicians in different locations could play together with greater ease when they focused on the shape of phrases, compared to when they focused on smaller divisions of temporal alignment (Chafe 2009). Although playing with longer musical phrases is also a strategy for musicality in Western classical music performance, telematic performance can bring new aesthetic possibilities of a shift of performance focus from smaller to larger temporal intervals.

The speed of light is faster than the speed of sound and visual information precedes auditory information in the natural world. Yet in network performance, this order is reversed (Chafe 2009). We hear the effect of a performer's action before we see the action. The relevance of cause and effect is explored further in digital artworks such as the Weblogmusic platform (Morris n.d.), a web-based venue for time-shifted improvising ensembles. In instance, we further explore the agency of performers in the telematic medium and express the disconnect between intention and effect with an African improvisation model towards aesthetic synchronicities and juxtapositions.

\subsection{Iterative design and composition process}

The telematic framework facilitates a data pipeline, inline with the specification of low-technical requirements on the dancer's side. Building from the foundation of the telematic framework, the iterative design process that we clarify in this section focuses on the sonic exploration of the music-dance interaction in the telematic DMDIs in instance. Our design interest lies in the embodied experience of both performers. Accordingly, we use soma-design strategies (Höök et al. 2018) within the RtD model (Zimmerman, Forlizzi and Evenson 2007).

\subsubsection{Research through Design Model}

The RtD model aims to draw the benefits of design practice into interaction design research in Human Computer Interaction (HCI). Zimmerman et al. (2007) propose four critical elements for evaluating RtD:

- Research should follow a clear, reproducible process.

- Research should comprise a significant invention that contributes to progress in the field.

- The work should provide extensibility, where the community can make use of the process in future design, or implement insights gained from the designed artefact.

- Research should be relevant.

According to this RtD model, the importance of validity through reproduction is shifted from the resulting artefact to the process, while artefacts are framed as 'concrete embodiments of theory and technical opportunities' (Zimmerman et al. 2007). The conceptualisation and dissemination of instance takes the perspective of RtD.

\subsubsection{Soma-design strategies}

The somaesthetic framework in philosophy combines 'soma' (relating to the body) and 'aesthetics' (relating to the appreciation of beauty) (Shusterman 2008). Somatic practices are applied in the dance community (Burnidge 2012; Araújo and Hein 2019) and the music community (Buchanan 2014; Noulis 2014). Soma-design practice uses somatic engagement to 
study the embodied experience of designers and users to inform the iterative design process. Soma-design principles can be particularly useful in interdisciplinary design research (Höök et al. 2018) and when designing 'new interfaces for musical expression' (Jensenius and Lyons 2017), such as DogDog (Bigoni and Erkut 2020). These principles are increasingly adopted in the HCI community (Palacio and Bisig 2017; Turmo Vidal and Márquez Segura 2018; Balaam et al. 2019; Eriksson, UnanderScharin, Trichon, Unander-Scharin, Kjellström and Höök 2019; Peng and Tanaka 2019; Umair, Alfaras, Gamboa and Sas 2019; Mainsbridge 2020). Somaesthetics and embodiment strategies can be useful to study telematic performance and are central to the design process of telematic performance works such as INTIMAL (Diaz et al. 2019). Since instance is an interdisciplinary project that combines embodied music, dance, telematic performance and sonic arts, the design process for sonic material centres on somatic principles.

The iterative design process for instance uses strategies under the embodied sketching umbrella, identified by Höök et al. (2018). We also used music and dance improvisation in three contexts: as a design tool to test iterations, as a method of composition and later in our performance practice. The processes for design and composition were symbiotic, where sonic and movement material developed alongside the DMDI.

\subsubsection{Iterations towards DMDIs}

In the iterative design process for the instance DMDIs, we first used a combination of bodystorming and physical movement sketching activities under the embodied sketching umbrella (Höök et al. 2018) to elicit a collection of movement qualities that the telematic performance space could evoke, with reference to the conceptualisations in section 4.1. Overall, this iteration produced three distinct movement qualities. Granularity evoked swift, punctuated movements in quick succession, in contrast to the light and breath-like characteristics of the other two movement qualities, suggestive of the ephemerality of the telematic performance space: the directionality of time brought continuous, floating qualities to mind; and the metamorphic process of fragmentation and transformation through digitalisation produced oscillatory, breathing qualities of dilation and contraction.

In the previous section, we elaborate on the association between gestural and visio-spatial mental imagery and the embodied experience of temporal navigation for musicians during performance. In the iterative process, the use of gestural mental imagery bridged an organic connection between conceptualisations on time in the telematic medium and sonic explorations. Inspired by our collection of movement qualities, we first constructed acoustic sound

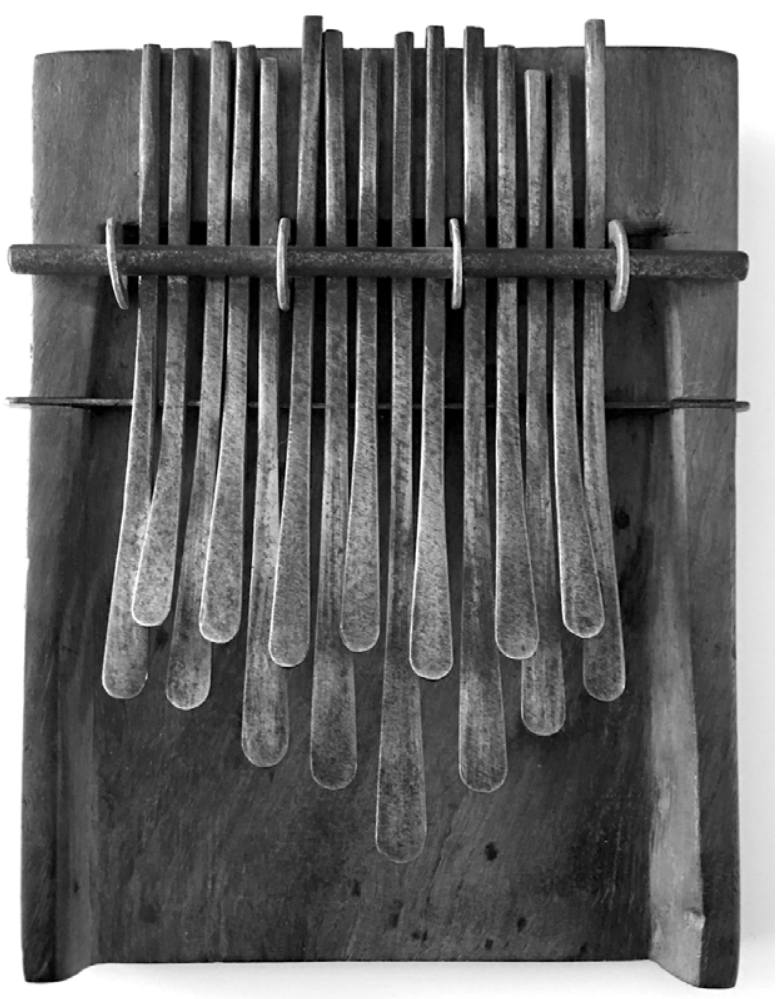

Figure 3. The mbira sampled in instance was made by master mbira player and maker Edgar Bera (Giminez 2018).

vocabularies of viola and mbira (Figure 3) to lay a foundation for the subsequent electroacoustics. We refined the design of the DMDIs in viola improvisation sessions, then in music-dance improvisation sessions.

To establish an acoustic sound vocabulary for the viola, the violist translated gestural imagery to musical affects by recording several short sonic sketches in a fluid, improvisatory process grounded in the embodied movement qualities described previously. The gestural imagery relating to granularity inspired pizzicato motifs and short, non-pitched sounds, such as tapping and bowing on the tailpiece and body of the instrument. When applied to arco techniques, this imagery evoked abrupt changes in bow acceleration. The continuous, floating gestural imageries elicited sustained lines of harmonics played with consistent bow speeds and slow, cyclical patterns. Inspired by the imagery of dilation and contraction, the violist explored the extremities of viola pitch range using overpressure to create subharmonics and playing on and behind the bridge to produce squeaks and scratches of varying lengths in the extreme high register. She smoothed the transitions between pitch ranges by varying bow speed, pressure and sound-point with each bow direction change to create oscillatory effects. By improvising, the violist discovered natural 


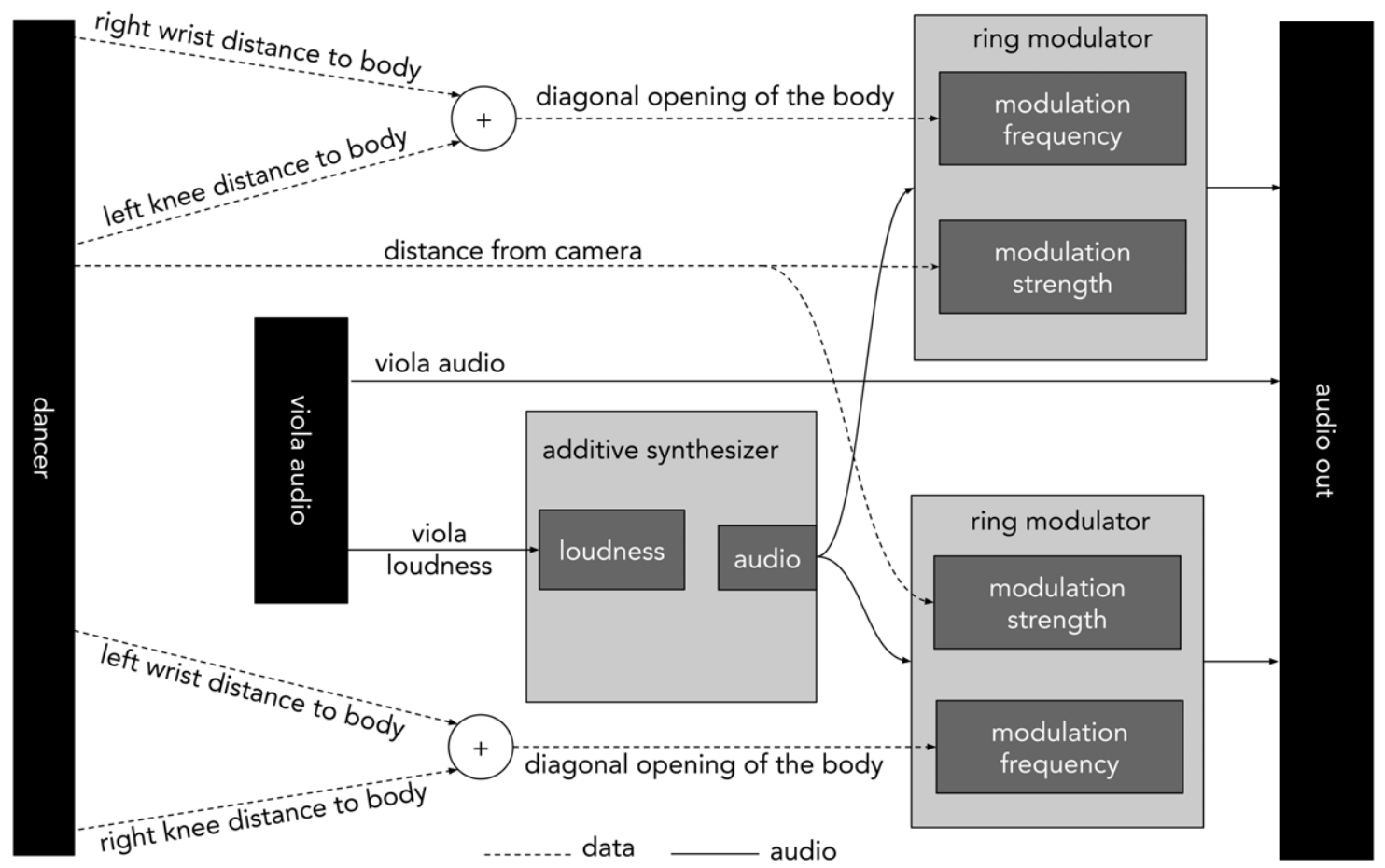

Figure 4. Additive synthesiser - Scenario 1.

groupings of techniques towards a strongly contrasting selection of sketches, which later guided the design of electroacoustic elements in instance.

The dancer - a mbira player himself - composed and recorded a short piece on mbira with a continuous, steady pulse, which comprises variations on an interlocking pattern, each variation highlighting different elements of an underlying melody that weaves between the interlocking parts. These characteristics are intrinsic to many styles of African music and are touched on in section 2.2. The cyclical structure of this composition also alludes to the oscillatory and cyclical breathing qualities, while notes plucked on mbira keys are naturally reminiscent of swift, punctuated movements in quick succession. In addition to the composition inspired by gestural imagery, we sampled each mbira key and captured the resonance of the instrument.

Before exploring the electroacoustic possibilities of the collection of affects, we tested the interactive qualities in improvised acoustic telematic music-dance sessions. The dancer's contribution as a somatic connoisseur (Höök et al. 2018) helped to refine the expression of musical affects through movement, defining and shaping the sonic aesthetics of instance. The dancer's agency over sonic material is an African concept, detailed in section 2.2. Since the sessions centred on dynamic qualities in relation to expressed meaning, this iteration is a choreography of interaction activity (Klooster and Overbeeke 2005) under the Embodied Sketching umbrella in soma-based design (Höök et al. 2018).
Having refined an acoustic vocabulary, we explored audio processing and synthesis techniques to create a prototype collection of three DMDIs, as described in the following. In our initial investigation into the electroacoustic possibilities of the mbira material, we created a digital version of the mbira using the sampled keys. We analysed one of the samples, and used the frequencies and amplitudes of the ten loudest partials from the sample's spectrogram to re-synthesise the sound through additive synthesis. Drawing from the continuous, floating imagery, we used the synthesised sound as a drone in the composition, transcribing the metallic timbre of mbira into the digital realm, outside its framework of discreet pitch constraints in the natural world. This material is implemented in DMDI 1 (Figure 4).

The first DMDI comprises a synthesised drone sound constructed from the timbre of mbira and a ring modulator in serial connection. The loudness of this additive synthesiser is mapped to the loudness of the viola input. The dancer adds timbral manipulations to the mbira drone sound by controlling two ring modulators for left and right channels where the diagonal expansion of his body controls the modulator frequencies. The sum of left wrist to body distance and right knee to body distance controls the modulator frequency of the ring modulator on the left channel. The mirror image mapping controls the modulator frequency of the ring modulator on the right channel. We mapped modulator strengths of both channels to the dancer's $z$-axis. This mapping scheme allows the violist and the dancer to control different parameters 


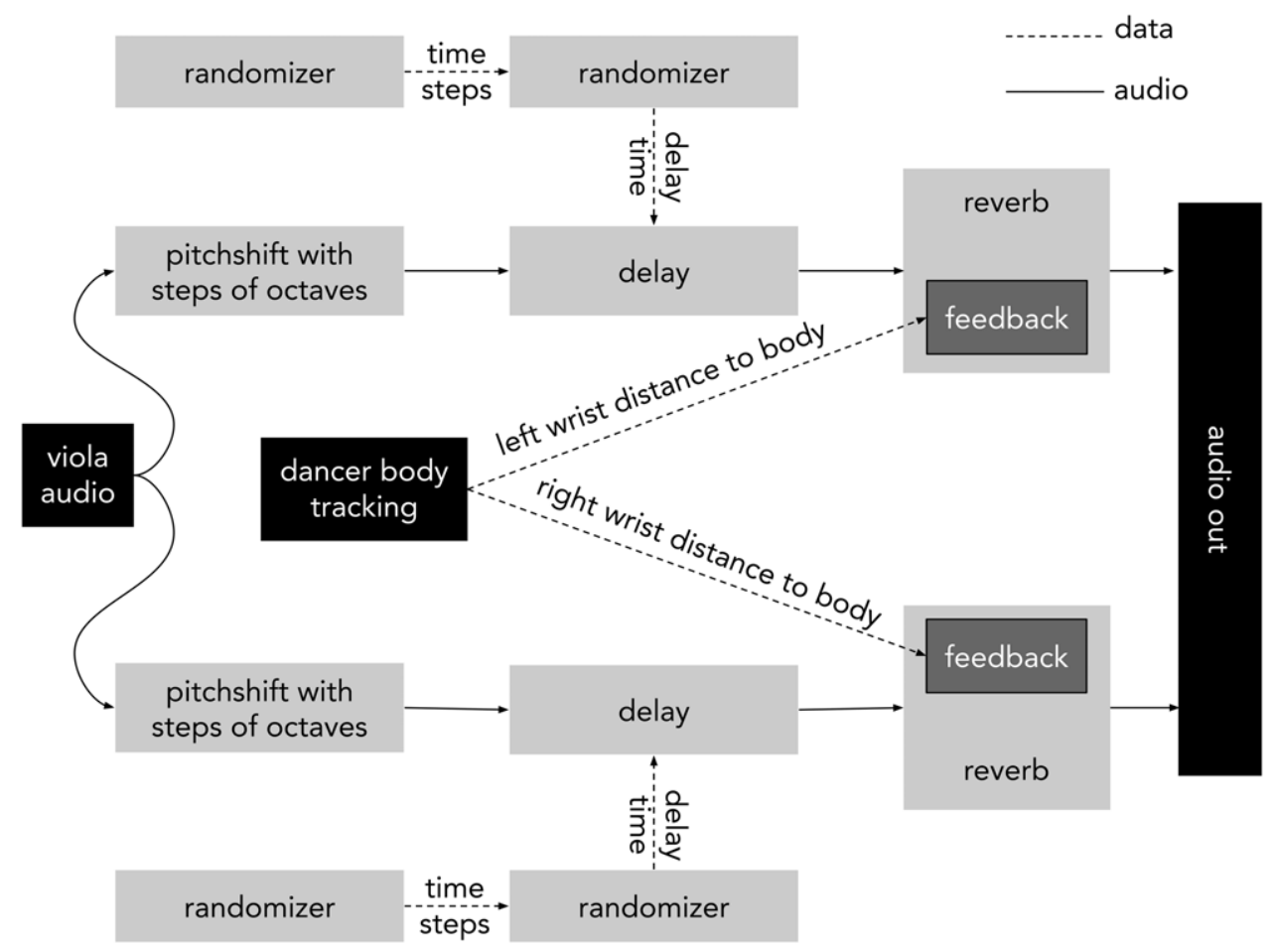

Figure 5. Granular synthesiser - Scenario 1 .

of one audio synthesis pipeline, while influencing the drone sound together.

Inspired by the dancer's swift, punctuated movements in quick succession, the second DMDI takes the form of a granuliser that processes live viola audio with chaotic elements in delay time (Figure 5). When paired with pizzicato motifs and short, non-pitched sounds, the granuliser produced discontinuous, erratic clusters of movement, which is in contrast to thecontinuous texture that was conjured with expansive and cumulative phrases when the granuliser was paired with sustained harmonics (Sound Examples 1 and 2). This DMDI adds polyphonic elements to the composition, in contrast with the drone sound generated by the additive synthesiser in DMDI 1 . The dancer controls reverb feedback amounts of both channels independently, using the distances of his left and right wrists to his body, respectively. This allows a different interaction strategy where the dancer controls one dimension of viola audio processing; thus, both performers create a single sound output together.

The expanding and contracting gestural imagery inspired spectral transformations with ring modulation and set in motion an exploration of time-based manipulations on the dancer's mbira composition recording, which is incorporated in DMDI 3 (Figure 6). This DMDI employs an audio player that is further processed by a ring modulator in serial connection, using the mbira audio material composed by the dancer. Additionally, the bow acceleration feature of viola controls the modulator frequency of the ring modulator. The audio output of this DMDI is demonstrated at 6 minutes 20 seconds into the recording (Video Example 1).

The dancer manipulates playback speed of the sample by expanding and contracting his body. The speed is mapped to positive and negative values to enable playback forward through the sample and in reverse. When the dancer moved in such a way that the sample was extremely slow, he could control each note as an event in time. This mapping strategy enabled an expression of ambiguity in the directional flow of time. The dancer explores the ambiguity of the directional flow of time at 7:07 in Video Example 1.

\section{END PRODUCT: GESTALT}

The textural sonic qualities and compositional structure of instance emerged from the combination of sonic and movement vocabularies with the electroacoustic material. The electroacoustic material is implemented in three DMDIs and the compositional structure comprises two sections. The first scenario introduces the performance space. The performers create a metaphorical sound space together by triggering and moulding a metallic drone sound: a figurative resonance reconstructed from the timbre of mbira (Figure 3). The sound, made up of its partials (section 4.2.3), implies the shape of a room by bringing its modes to light. Over three minutes, the pitch of the drone falls: the metaphorical space expands and at 


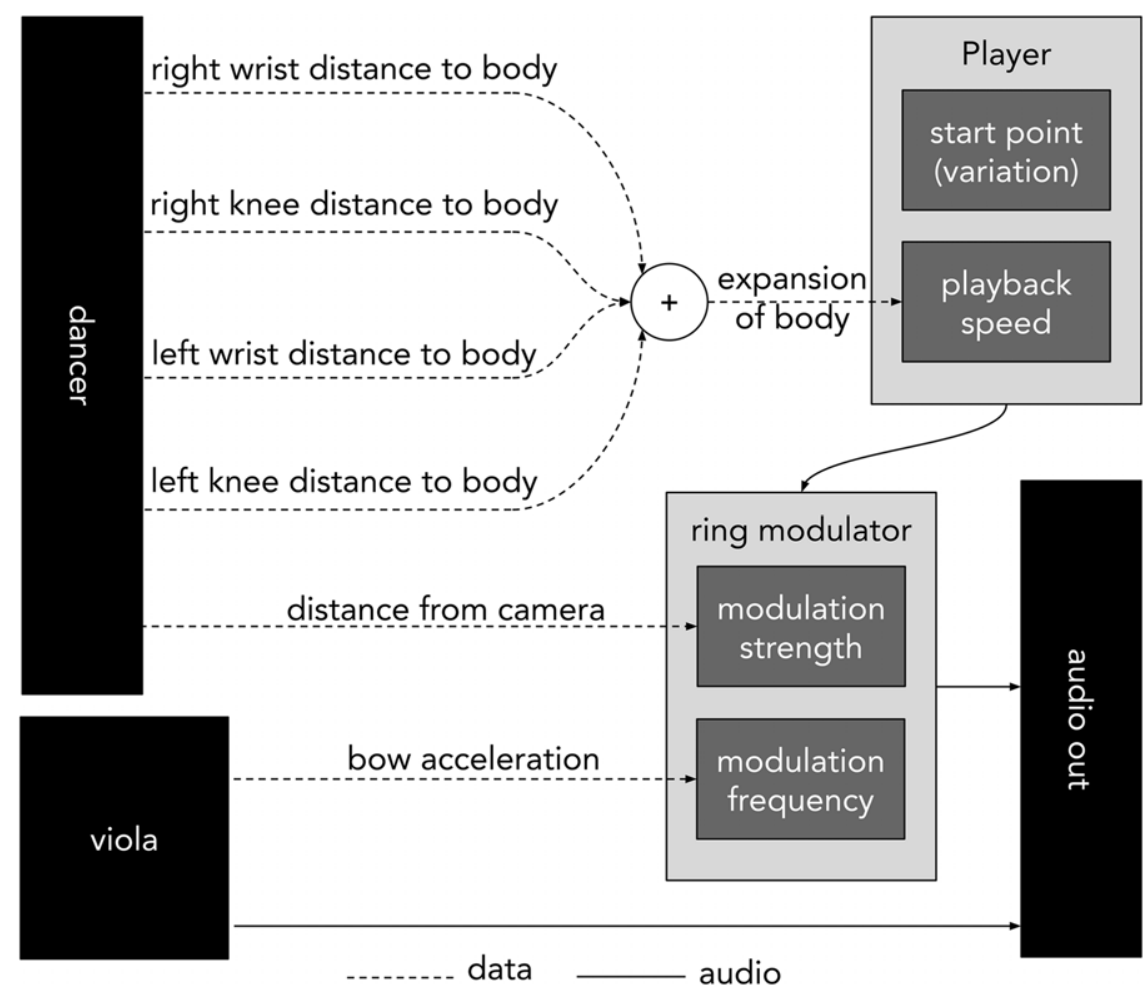

Figure 6. Sample playback and processing - Scenario 2.

the same time the performers settle into the digital performance space. In this scenario, we hear the timbre of mbira in the digital realm outside its framework of discreet pitch constraints in the natural world.

In the second scenario, the performers explore their individual and combined interactions with time, as it behaves in the digital, telematic performance space. Using audio manipulations in the time domain, the performers fall in and out of an experience of pulse. Similar to music-dance interactions in the Baganda Baakisimba practice (section 2.2), the performance slips in and out of pulse through intentional and surprising moments of gestural alignment, pointing to the elusive nature of time in the telematic medium (section 4.1).

Scenario 2 begins 4 minutes and 48 secs into the performance (Video Example 1). Here, the multi-user instrument centres around a specific audio sample composed for and played on mbira by the dancer. The piece is transcribed from the single-user mbira to the multiuser DMDI: an allusion to de- and reconstruction through fragmentation in telematic performance (section 4.1). The audio recording has a continuous, steady pulse and comprises variations on an underlying pattern. The dancer switches between variations as he moves along the $x$-axis of the screen. The sonic output of this mapping is evident at 5:12 in the performance recording (Video Example 1). In contrast with the steady pulse, the dancer also performs live alterations to the sample in the time domain to explore the performance in an ambiguous present moment caused by network delay.

\section{THE PROCESS AND EXPERIENCE}

Having used a soma-design approach, detailed in sections 3.3.2 and 3.3.3, the discussion is also based on somaesthetic principles to reflect on the performers' resulting embodied experience in their multimodal interaction. The rehearsals provided insights to make the final DMDI iterations before the public performance of instance, and the performance provides insights for future performances using the DMDI.

\subsection{Rehearsals}

In the preparation, the performers used a combination of soma-based principles, informal discussion sessions and concepts in the African performing arts to play with the idiosyncrasies of the telematic medium.

Rehearsal sessions for instance began when the DMDIs were still being conceptualised, and were intrinsic to both prepared and improvised compositional processes. Early rehearsals took the form of instant-composition play sessions on Zoom, where the performers used soma-based processes to guide the performance interaction, both with and without the DMDIs, identifying peculiarities and challenges of performance practice in the telematic medium. 
One of the play sessions without the DMDI is documented in Video Example 2.

At first, the performers struggled to find a sense of flow, producing unintentional pauses from the delay of action and reaction due to latency, which was between 100 and $150 \mathrm{~ms}$ due to Zoom video connection, well above the 'edge of playability' for rhythmically synchronised musical duos of 55 to 66 ms described by Chafe, Cáceres and Gurevich (2010). This was particularly evident when playing with shorter gestures and in moments when they were each unsure if the effect of their actions had been successfully portrayed to the other performer. In addition, the motivations behind their gestures were often constrained by the technology rather than by artistic decisions. As a result, they used less pause than when practising music-dance outside the telematic medium, reflecting that they often produced sound and movement simply to test whether sound and movement could still propagate through the network. The performers experienced both of these peculiarities as indications of a lack of trust, between each other, and in technology too.

To build a foundation for an embodied empathetic interaction towards flow, the performers held informal discussion sessions before and after each rehearsal. Conversation flowed freely between topics related to telematic performance, and topics related to day-today life. Without the luxury of playing together in the same space, verbal communication helped the performers to construct contexts around each other. Alongside music-dance rehearsals, they perceived these discussions as a necessary contribution to building rapport, towards the perception of a shared creative experience of flow when playing together.

As the development of gestural and musical affects progressed during the iterative design process (section 4.2.3), the dancer noticed changes in the violist's telematic improvisation, in comparison to when they had played together in the same physical space. Musical transitions were smoother and more gradual, indicating a shift of focus from smaller temporal divisions to larger ones and from a focus on individual gestures to an overall sonic output. The violist was improvising musical compensations for network latency by altering the way she communicated expression of emotional affect through time, which is in line with the observations of the network music trial between Norway and the United States, mentioned in section 1 (Chafe 2009). The violist's temporal adjustment also reflects a shift of focus from 'what is' to the embodied human experience; as discussed in section 2.2, Rovelli (2019) writes that we might experience space-time to flow continuously only because the limitations of the human interface with reality do not allow us to distinguish the smallest temporal intervals.
Though the performers could lengthen their phrasing to adjust to network latency, they still experienced ambiguity of cause and effect in the telematic medium (section 4.1). To explore solutions, the performers improvised with a variety musical qualities (section 3.3.3). Playing in slow tempi with longer notes and smoother gestures, the performers felt they could keep track of action and reaction more easily than when using more pause and playing shorter notes and gestures. However, maintaining a sense of flow became easier when they each focused on their individual contributions to their exchange of musical and gestural quality and character, rather than on any definitive composition of sound and gesture in their shared sonic output. Here, the performers played with African performance principles in mind, embracing perceptual shift and unintended synchronicities (section 2.2) to explore the effect of fragmentation on the order of time in the telematic medium (section 4.1).

Although performers' focus on a shared sonic output is a defining feature of a multi-user instrument (Jordà 2005), it is equally important that performers embody concepts of perceptual shift within their individual experience, when the multi-user DMDI is used in the telematic medium. During rehearsals, the strategies used to transfer music-dance practice to the telematic medium were simultaneously used to learn to play the DMDIs, as a result of the resemblance of familiar instruments (outlined in section 3.3.2) in the DMDI design.

\subsection{Performance}

The performance discussion explores the impact of the addition of an audience on the performers' experienced interaction with the DMDIs, the performance being the resulting artefact from the iterative design process inline with RtD approach (section 3.3).

Towards the design specification of minimal technical set-up for the dancer, the musician's laptop handles all computational processing. The dancer's video stream is used for the DMDI controls, for visual feedback to the violist and for the YouTube stream to the audience (Figure 1). This asymmetrical design leads to contrasts in the performance experiences of the two performers, described in the following.

Although the violist played the DMDIs through playing her viola, she was in charge of running the telematic framework and preparing and triggering the compositional scenarios (section 4.2.3) on her laptop. She experienced these brief interactions with her laptop as abrupt breaks in her experience of flow. One such moment occurs at 4 minutes in the video of the public performance (Video Example 1). To play around these distractions during the performance, she used a strategy similar to the change in interest 
soma-design strategy described by Höök et al. (2018) by turning away from the camera and the video-feed of the dancer to focus her attention inward: first to her own body, taking note of her breathing, then gradually moving outward to her muscles, then to the points of contact between her body and the viola. By drawing her attention inward to her own body, she re-centred herself. From focusing on her embodied experience of the familiar interaction with the viola, she shifted her somatic awareness outward, towards an empathetic connection with the dancer.

When performing Scenario 1, the performers became frustrated that their input to play the multiuser instrument was imbalanced. The mapping design dictated that the violist had to trigger all the electroacoustic material. When she stopped playing, the sonic expression of the dancer's gestural input disappeared. Although this imbalance could potentially facilitate an artistic exploration of balance in agency, a solution for future performances would be to map one of the dancer's controls to the loudness of the drone sound, so that his movements can produce sound when the violist is not playing. Although the dancer moved closer and further from the camera in rehearsals, he positioned himself at his maximum possible distance from the camera throughout Scenario 2 in the performance. His decision meant that his whole body was visible to the camera, allowing for precision in his DMDI controls through PoseNet gesture detection (section 3.2), yet it also resulted in a lower ring modulation strength (section 5). An audience member with no prior knowledge of the mapping design would be completely unaware of the DMDI's full potential for timbral manipulation through ring modulation, including the musician's ability to affect ring modulator frequency with her bow acceleration and the dancer's ability to control the DMDI with his distance from the camera. The violist also found herself resisting the urge to demonstrate all the possibilities of each DMDI in a short space of time, using the comparison that when she plays acoustic improvised or Western classical music, she does not play every possible pitch and tone quality on her viola, so perhaps it is not necessary to expose every sonic possibility of a DMDI during performance.

The performers reflected on the multimodality of their gestures. During the performance, gestures served three functions simultaneously: to control the DMDIs, to contribute to the aesthetic performance output for the audience and to communicate with each other. The multiplicity of gestural effect was experientially different for the musician and dancer because of the nature of their different modes of expression. While the dancer's movements were naturally intended as both performative and communicative through dance, the violist conceptualised her gestures first as necessary for the production of sound and then as performative.

The telematic interface in instance dictated that communicative and performative gestures were by default perceived by the audience as performative gestures, where any small gestures intended as visual cues were equally visible to the audience and had an effect on the shared sonic output. As a result of this design framework, the violist had no more visual or sonic feedback from the dancer than the audience, and experienced a negative impact on her rapport with the dancer as a result of the addition of the audience in the performance setting, compared to her experience in rehearsals.

Audio transferred through a network can naturally exist throughout another space, while movement transferred through video material is bound to the constraints of a screen. In addition to the audio feedback from the dancer's input to the DMDIs, the musician relied heavily on visual feedback from the dancer's video material. The dancer relied more on sonic feedback than visual feedback from the musician and could move freely about the space, without the need for a conscious interaction with technology to feel connected with the musician. With a simple technical set-up, the dancer felt that he could focus on his somatic experience in a similar way to the rehearsal context.

\section{CONCLUSION}

The design, composition and rehearsal processes for instance were symbiotic and ran simultaneously. We combined technical concepts within multi-user and music-dance instruments to create a series of networked DMIs, drawing inspiration from the body for sonic expression and reflection on philosophical and somatic concepts in the telematic medium. With a soma-design approach, we incorporated the DMIs into a DMDI interface, which neither performer had to physically touch while playing, bringing to light themes of ephemerality and disembodiment in the telematic performance space.

The performers used improvised music-dance play sessions to test the design of instance and to play into juxtapositions within collaborative music-making practices. Using a digital multi-user instrument in the telematic performance space required the performers to simultaneously play towards one combined sonic output and to embrace their individual embodied experience of flow and perceptual shift.

The telematic medium brings the asymmetries in music-dance interaction to light. Through a public performance, the performers reflected on the impact of the audience on the embodied experience of musicians and dancers in the telematic performance 
space. They found the nature of this impact to be affected by the mode of feedback from their coperformer, where the dancer relies on sonic feedback and the musician relies on visual feedback and the telematic propagation of sonic and visual performance elements.

As an artwork, instance is by no means finished and lends itself undergo further design iterations according to the insights gained from each performance. It provides an African philosophical and somatic starting point to develop an embodied multimodal performance practice in the telematic performance space, using somaesthetic principles in African performance to play through the ambiguous order of time.

\section{Acknowledgements}

We thank Prof. Marina Thibeault and Dr Robert Pritchard at the University of British Columbia and Paul Rommelaere at the University of Cape Town for their insights. We would also like to thank the Social Sciences and Humanities Research Council of Canada and the Oppenheimer Memorial Trust for funding this research.

\section{SUPPLEMENTARY MATERIAL}

To view supplementary material for this article, please visit https://doi.org/10.1017/S1355771821000479

\section{REFERENCES}

Agawu, K. 2014. Representing African Music: Postcolonial Notes, Queries, Positions. Abingdon: Routledge.

Araújo, M. V. and Hein, C. F. 2019. A Survey to Investigate Advanced Musicians' Flow Disposition in Individual Music Practice. International Journal of Music Education. 37(1): 107-17.

Balaam, M., Comber, R., Clarke, R. E., Windlin, C., Ståhl, A., Höök, K. and Fitzpatrick, G. 2019. Emotion Work in Experience-Centered Design. Proceedings of the 2019 CHI Conference on Human Factors in Computing Systems, Glasgow, Scotland, 1-12. https://doi.org/10. $1145 / 3290605.3300832$

Berliner, Paul F. 2020. The Interlocking Aesthetic. The Art of Mbira. University of Chicago Press, 81-8.

Bigoni, F. and Erkut, C. 2020. DogDog: Soma-Based Interface Design for an Improvising Musician. Proceedings of the 7th International Conference on Movement and Computing, Jersey City, NJ, 1-4. https://doi.org/10.1145/3401956.3404242

Bischoff, J., Gold, R. and Horton, J. 1978. Music for an Interactive Network of Microcomputers. Computer Music Journal 2(3): 24. https://doi.org/10.2307/3679453

Buchanan, H. J. 2014. Body Mapping: Enhancing Voice Performance Through Somatic Pedagogy. In S. D. Harrison and J. O'Bryan (eds.) Teaching Singing in the 21st Century. Dordrecht, Netherlands: Springer, 143-74. https://doi.org/10.1007/978-94-017-8851-910

Burnidge, A. 2012. Somatics in the Dance Studio: Embodying Feminist/Democratic Pedagogy. Journal of Dance Education 12(2): 37-47. https://doi.org/10.1080/ 15290824.2012 .634283

Cage, J. 1960. Imaginary Landscape, No. 4 (March No. 2). CF Peters. www.edition-peters.com/product/imaginarylandscape-no-4-march-no-2/ep6718a (accessed 11 November 2021).

Chafe, C. 2009. Tapping into the Internet as an Acoustical/ Musical Medium. Contemporary Music Review 28(4-5): 413-20. https://doi.org/10.1080/07494460903422362

Chafe, C., Cáceres, J. P. and Gurevich, M. 2010. Effect of Temporal Separation on Synchronization in Rhythmic Performance. Perception 39(7): 982-92. https://doi.org/ 10.1068/p6465

de Cheveigné, A. and Kawahara, H. 2002. YIN, a Fundamental Frequency Estimator for Speech and Music. The Journal of the Acoustical Society of America 111(4), 1917. https://doi.org/10.1121/1.1458024

Diaz, X. A., Boddie, P., Erdem, C., Aandahl, E., Andersen, E. S., Dahl, E., Lesteberg, M. and Jensenius, A. R. 2019. Sensing Place and Presence in an INTIMAL LongDistance Improvisation. Journal of Network Music and Arts 1(1): 24.

Erdem, C., Schia, K. H. and Jensenius, A. R. 2019. Vrengt: A Shared Body-Machine Instrument for Music-Dance Performance. Proceedings of the International Conference on New Interfaces for Musical Expression, Porto Alegre, Brazil, 186-91. https://doi.org/10.5281/zenodo.3672918

Eriksson, S., Unander-Scharin, A., Trichon, V., UnanderScharin, C., Kjellström, H. and Höök, K. 2019. Dancing with Drones: Crafting Novel Artistic Expressions Through Intercorporeality. Proceedings of the 2019 CHI Conference on Human Factors in Computing Systems, Glasgow, Scotland, 1-12. https:// doi.org/10.1145/3290605.3300847

Höök, K., Caramiaux, B., Erkut, C., Forlizzi, J., Hajinejad, N., Haller, M. et al. 2018. Embracing First-Person Perspectives in Soma-Based Design. Informatics 5(1): 8. https://doi.org/10.3390/informatics5010008

Jensenius, A. R. and Lyons, M. J. (eds.) 2017. A NIME Reader: Fifteen Years of New Interfaces for Musical Expression. Cham, Switzerland: Springer International.

Jordà, S. 2005. Multi-user Instruments: Models, Examples and Promises. Proceedings of the International Conference on New Interfaces for Musical Expression, Vancouver, BC, Canada, 23-6. https://doi.org/10.5281/zenodo.1176760

Klooster, S. and Overbeeke, C. J. 2005. Designing Products as an Integral Part of Choreography of Interaction: The Product's Form as an Integral Part of Movement. Design and Semantics of Form and Movement, Proceedings of the 1st European Workshop on Design and Semantics of Form and Movement, Newcastle, UK, 23-35.

Kubik, G. 1969. Composition Techniques in Kiganda Xylophone Music: With an Introduction into some Kiganda Musical Concepts. African Music: Journal of the African Music Society 4(3): 22-72. https://doi.org/ 10.21504/amj.v4i3.1437 
Lemmon, E. C. 2019. Telematic Music vs. Networked Music: Distinguishing Between Cybernetic Aspirations and Technological Music-Making. Journal of Network Music and Arts 1(1): 30. https://commons.library. stonybrook.edu/jonma/voll/iss $1 / 2$

Locke, D. 2009. Simultaneous Multidimensionality in African Music: Musical Cubism. African Music: Journal of the International Library of African Music 8(3): 8-37. https://doi.org/10.21504/amj.v8i3.1826.

Mainsbridge, M. 2020. Soma-based Non-Physical Instrument Design in Electronic Music Performance. Leonardo, 1-8. https://doi.org/10.1162/leona01883

Morris, J. M. 2015. Weblogmusic: A Performance Platform for Ensembles of Individually Time-Shifted Improvisers. Proceedings of the 21st International Symposium on Electronic Art.

Muller, P., Burger, B., Giovanni, J. D. and Ziegler, M. 2019. Understanding the Telematic Apparatus. Journal of Network Music and Arts 1(1): 27.

Nannyonga-Tamusuza, S. 2015. Music as Dance and Dance as Music: Interdependence and Dialogue in Baganda Baakisimba Performance. Yearbook for Traditional Music 47: 82. https://doi.org/10.5921/yeartradmusi.47.2015.0082

Noulis, C. 2014. Somatic Education and Piano Performance. Doctoral diss., Birmingham City University, UK. www.open-access.bcu.ac.uk/4860/ (accessed 29 November 2020).

Palacio, P. and Bisig, D. 2017. Piano\&Dancer: Interaction Between a Dancer and an Acoustic Instrument. Proceedings of the 4th International Conference on Movement Computing - MOCO '17, Goldsmiths, University of London, UK, 1-8. https://doi.org/10. 1145/3077981.3078052

Pardue, L. S., Buys, K., Edinger, M., Overholt, D. and McPherson, A. P. 2019. Separating Sound from Source: Sonic Transformation of the Violin Through Electrodynamic Pickups and Acoustic Actuation. Proceedings of the International Conference on New Interfaces for Musical Expression, Porto Alegre, Brazil, 6.

Peng, Y. F. and Tanaka, A. 2019. Body and Embodiment in Dance Performance. Proceedings of the 6th International Conference on Movement and Computing, Tempe, AZ, USA, 1-6. https://doi.org/10.1145/3347122.3359596

Rovelli, C. 2019. The Order of Time. New York: Riverhead Books.

Schnell, N., Röbel, A., Schwarz, D., Peeters, G. and Borghesi, R. 2009. MuBu and Friends - Assembling Tools for Content Based Real-Time Interactive Audio Processing in Max/MSP. Proceedings of International Computer Music Conference (ICMC), Montreal, Quebec, Canada.

Schnell, N., Schwarz, D., Larralde, J. and Borghesi, R. 2017. PiPo, a Plugin Interface for Afferent Data Stream Processing Modules. Proceedings of International
Symposium on Music Information Retrieval (ISMIR 2017), Suzhou, China, 8. https://hal.archives-ouvertes. $\mathrm{fr} /$ hal-01575288/file/SchnellEtAl\%20-ismir2017-\% 20pipo.pdf

Schoenberg, A. 1952. My Evolution. The Musical Quarterly 38(4): 517-27.

Shusterman, R. 2008. Body Consciousness: A Philosophy of Mindfulness and Somaesthetics. Cambridge: Cambridge University Press.

Stachó, L. 2018. Mental Virtuosity: A New Theory of Performers' Attentional Processes and Strategies. Musicae Scientiae 22(4): 539-57.

Tatar, K. 2021. Ktatar/String-Feature-Extraction. https:// github.com/ktatar/string-feature-extraction (accessed 16 January 2021).

Tatar, K. and Pasquier, P. 2019. Musical Agents: A Typology and State of the Art Towards Musical Metacreation. Journal of New Music Research 48(1): 56-105. https://doi.org/10.1080/09298215.2018.1511736

Tragtenberg, J., Calegario, F., Cabral, G. and Ramalho, G. 2019. Towards the Concept of 'Digital Dance and Music Instrument'. Proceedings of the International Conference on New Interfaces for Musical Expression, Porto Alegre, Brazil, 6.

Turmo Vidal, L. and Márquez Segura, E. 2018. Documenting the Elusive and Ephemeral in Embodied Design Ideation Activities. Multimodal Technologies and Interaction 2(3): 35 . https://doi.org/10.3390/ mti2030035

Umair, M., Alfaras, M., Gamboa, H. and Sas, C. 2019 Experiencing Discomfort: Designing for Affect from First-Person Perspective. Adjunct Proceedings of the 2019 ACM International Joint Conference on Pervasive and Ubiquitous Computing and Proceedings of the 2019 ACM International Symposium on Wearable Computers, London, UK, 1093-6. https://doi.org/10. $1145 / 3341162.3354061$

Yogo, Y. 2021. Yuichkun/n4m-posenet. https://github.com/ yuichkun/n4m-posenet (accessed 14 January 2021).

Zimmerman, J., Forlizzi, J. and Evenson, S. 2007. Research Through Design as a Method for Interaction Design Research in HCI. Proceedings of the SIGCHI Conference on Human Factors in Computing Systems CHI '07, San Jose, CA, USA, 493-502. https://doi.org/ $10.1145 / 1240624.1240704$

\section{VIDEOGRAPHY}

Gimenez, L. 2018. Episode Five: How to Play Zimbabwean Mbiras by Edgar Bera. YouTube. (accessed 15 January 2021).

instance. 2020. https://lucystraussmusic.com/instance (accessed 15 January). 